Research Article

\title{
Bit Error Rate Analysis for FH/MFSK System with Follower Jamming
}

\author{
Xinfeng Fan (iD, Zhiliang Tan, and Peijiao Song \\ Army Engineering University, Shijiazhuang Campus, National Key Laboratory on Electromagnetic Environment Effects, \\ Shijiazhuang 050003, China \\ Correspondence should be addressed to Xinfeng Fan; 1132544091@qq.com
}

Received 27 December 2019; Revised 23 June 2020; Accepted 7 July 2020; Published 27 July 2020

Academic Editor: Xiangyu Meng

Copyright (c) 2020 Xinfeng Fan et al. This is an open access article distributed under the Creative Commons Attribution License, which permits unrestricted use, distribution, and reproduction in any medium, provided the original work is properly cited.

At present, methods of bit error rate (BER) analysis for frequency-hopping (FH) system can only solve the problem of barrage jamming, but these are no comprehensive means to follower jamming (FJ). This paper proposes a method of BER analysis with FJ based on frequency hopping M-ary frequency-shift keying (FH/MFSK) system. Through the analysis of the mechanism of FH/ MFSK system, assuming the wireless channel is an AWGN channel, the BER formulas for different jamming types are derived, respectively, from the point of one-dimensional probability density function of the output signal of the envelope detector, while judgment principle of envelope detecting method is used. Results show that the BER of multiple FH/MFSK system with FJ is relatively lower. In the worst case of FJ, the BER of FH/4FSK system and FH/8FSK system is reduced by $2 \mathrm{~dB}$ and $3 \mathrm{~dB}$, respectively, compared with that of FH/2FSK system. The method of BER analysis proposed provides an effective means to research the BER with FJ in depth.

\section{Introduction}

FH communication has a strong ability to resist jamming and multipath fading [1]; therefore, it has been widely used in tactical communication and civil use [2]. It is of great significance to study the change of BER for FH communication system after it is jammed and to take effective measures to improve the communication quality. FJ and barrage jamming are the main artificial jamming in $\mathrm{FH}$ communication. At present, the research about BER analysis for FH communication mainly focuses on barrage jamming, including broadband barrage jamming and narrow-band jamming and partial-band noise jamming (PBNJ). Duan and Zhong [3] analyzed the BER performance of PBNJ for multihop FH/MFSK by means of combination of all probability analysis and multivariate distribution. Zhang and Teh $[4,5]$ studied the composite effects of multitone jamming (MTJ) and PBNJ for maximum-likelihood fast FH/ MFSK receiver over Rayleigh-fading channels. Quan et al. $[6,7]$ put forward a multisequence frequency hopping system; they derived the BER formulas with PBNJ by using the decision criterion of choosing large size, and the BER performance is analyzed. Dong et al. [8] set the minimum resolution of broadband frequency sweeping jamming (BFSJ) bandwidth to $1 / M$ of the MFSK signal bandwidth for FH/MFSK and derived the closed-form expressions of BER.

From above, the existing research about the BER analysis for FH communication is mainly aimed at barrage jamming, while public research of BER quantitative analysis about $\mathrm{FJ}$ is partial but not comprehensive. Riddle [9] had analyzed the performance of a hybrid spread spectrum system against FJ. The error probability of a FH system with typical FSK modulation was derived in the absence of FJ. Though the error probabilities in different communication channels were gained, the modulation mode was of low order, and the jamming mode only considered narrow-band noise. Hassan et al. [10] had examined tone jamming for FH with M-ary FSK modulation and found that the two-tone jamming was the worst case scenario. BERs of different communication and jamming modes were gained from the point of jamming transmitting channel. The jamming signal only considered tone case similarly. 
FJ refers to a jamming mode whose jamming signal can follow frequency jump of $\mathrm{FH}$ signal [11]. Its characteristics are similar to $\mathrm{FH}$ signal in time domain and frequency domain, but their modulation information is generally different. The modulation signal of FJ can be noise, pulse, or single tone. Therefore, it needs to clear the FJ type before analyzing BER performance for $\mathrm{FH}$ system; different analytical strategies are adopted for different FJ types. Existing methods of BER analysis for $\mathrm{FH}$ system with barrage jamming actually assumed that the modulation signal of jamming is noise, and the jamming power spectral density (PSD) is superimposed with the external noise PSD. This processing way does not consider single tone and other modes, and it cannot be applied to BER analysis with FJ. Existing methods of BER analysis for FH system with FJ were not comprehensive, ignoring the jamming modes or higher modulation order.

Motivated by this, a method of BER analysis with FJ is proposed for FH/MFSK system, and theoretical BER formulas are established. The method is based on the judgment principle of envelope detecting method for two typical types of FJ. Simulation results are given to prove correctness of the theoretical derivation.

\section{System Model}

The principle block diagram of FH/MFSK system is shown in Figure 1 [12], mainly including the transmitting end and the receiving end. It describes the main signal processing steps. Compared with the practical system, it lacks the secondary steps such as RF filters, IF filters, and so on.

At the transmitting end, the input information is first modulated by MFSK modulator. IF modulated signal is mixed with the hopping RF signal synthesized by the frequency synthesizer to perform frequency shifting at $\mathrm{FH}$ modulator. The frequency of the resulting RF signal is constantly changing, and the anti-interception performance and antijamming performance are greatly improved. At the receiving end, the frequency is synchronized first according to the received signal. Then, the system mixes the received signal with the hopping RF signal output by the frequency synthesizer to obtain an IF modulated signal, performing MFSK demodulation by MFSK demodulator to obtain original information.

The low-pass equivalent signal of the MFSK signal can be expressed as

$$
s(t)=\sqrt{\frac{2 E_{s}}{T_{d}}} e^{j 2 \pi m \Delta f t}, \quad\left(m=1,2, \ldots, M, 0 \leq t \leq T_{d}\right),
$$

where $E_{s}$ is the signal symbol energy in the transmitting tone; $T_{d}$ is the frequency duration time; $\Delta f$ is the frequency interval of adjacent MFSK tone; and $M$ is the order of frequency shift-keying modulation.

The FH/MFSK signal can be expressed as

$$
x(t)=\sqrt{\frac{2 E_{s}}{T_{d}}} e^{j 2 \pi\left(m \Delta f+f_{n}\right) t},
$$

where $f_{n}$ is the frequency of the $n$th hop.
Assuming that there is no synchronization error at the receiving end, and the effects of amplitude attenuation and signal transmission delay are ignored, then the received signal can be expressed as

$$
r(t)=x(t)+J(t)+n(t)
$$

where $J(t)$ represents the FJ; $n(t)$ is the noise due to additive white Gaussian noise (AWGN) with zero mean and variance of $\sigma_{w}^{2}=\left(N_{0} / 2\right) B$, where $N_{0}$ is the PSD of the noise and bandwidth $B$ meets the condition $B=1 / T_{d}$ [13]. The mathematical model of follower jamming can be expressed as

$$
J(t)=\sqrt{2 P_{j}} m_{j}(t) e^{j 2 \pi\left(m \Delta f+f_{n}\right)(t-\Delta t)},
$$

where $P_{j}$ is the power and $m_{j}(t)$ is the modulated signal of FJ. In this paper, narrow-band noise and single tone signal are considered. $\Delta t$ is the time delay of FJ signal compared with $\mathrm{FH}$ signal at each frequency and meets the condition $0 \leq \Delta t<T_{d}$.

\section{BER Analysis}

It is assumed that the FH set contains $N$ frequency points, which are evenly distributed and the hopping speed is constant. For the convenience of analysis, it is also assumed that FJ forms total jamming to all frequency duration time with $\Delta t=0$. The transport channel is assumed to be AWGN channel. FH/MFSK signal is demodulated by envelope detection method as shown in Figure 2. The FH signal is equivalent to the fixed-frequency signal at each frequency point. Thus, the process of BER analysis for FH/ MFSK system is similar to that of BER analysis for MFSK system.

Error rate usually includes bit error rate and symbol error rate (SER), and bit error rate is discussed in this paper.

When there is no FJ but external noise alone, the BER of $\mathrm{FH} / \mathrm{MFSK}$ system is the same as MFSK system, given by [14]

$$
P_{b}=\frac{1}{2(M-1)} \exp \left(-\frac{l E_{b}}{2 N_{0}}\right) \sum_{i=2}^{M}(-1)^{i} C_{M}^{i} \exp \left(\frac{l E_{b}(2-i)}{2 N_{0} i}\right),
$$

where $l=\log _{2} M$ and $E_{b}$ is the signal bit energy.

3.1. Modulated Signal of FJ Is Narrow-Band Noise. When the modulated signal of FJ is narrow-band noise, called as follower noise jamming (FNJ), it can be modeled as a zero mean Gaussian random variable, whose PSD $\left(N_{j}\right)$ is evenly distributed across the hopping bandwidth. The variance can be expressed as $\sigma_{j}^{2}=\left(N_{j} / 2\right) B$.

It is assumed that the outputs of envelope detector are $V_{i}(i=1,2, \ldots, M)$ as shown in Figure 2, and the FH/MFSK signal is jammed by FJ at the first tone. Thus, the output of envelope detector 1 contains FJ and external noise simultaneously. $V_{1}$ is a generalized Rayleigh distribution with zero mean and variance of $\sigma^{2}$, and its one-dimensional probability density function can be expressed as [15] 


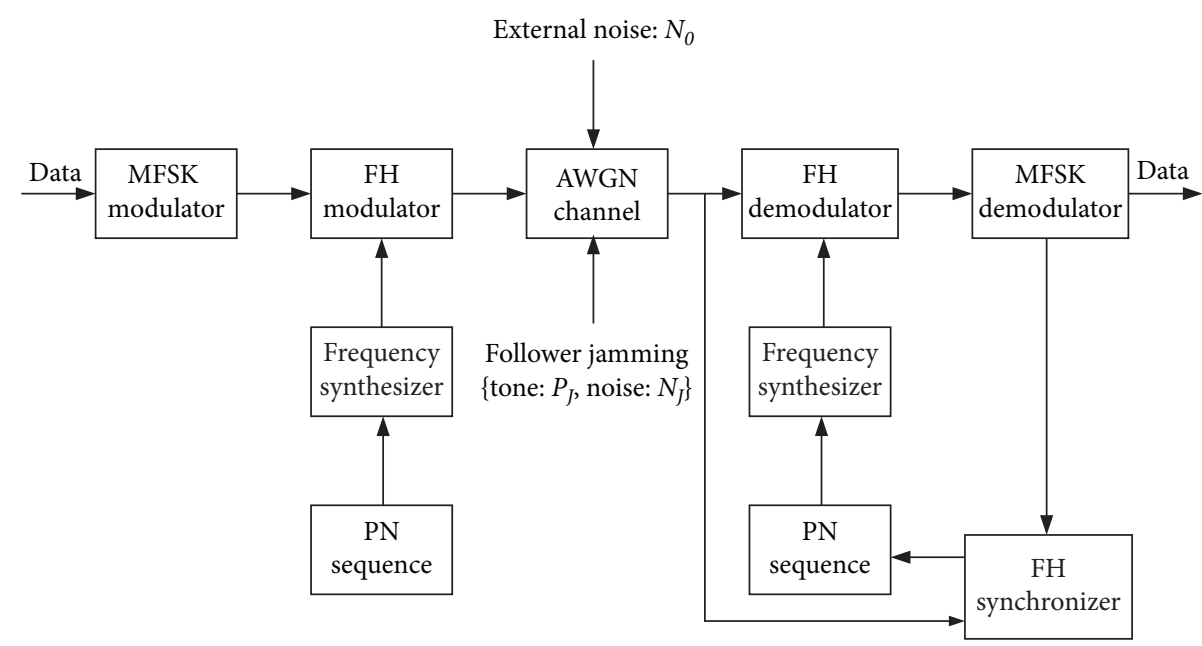

FIGURE 1: Principle block diagram of FH/MFSK system.

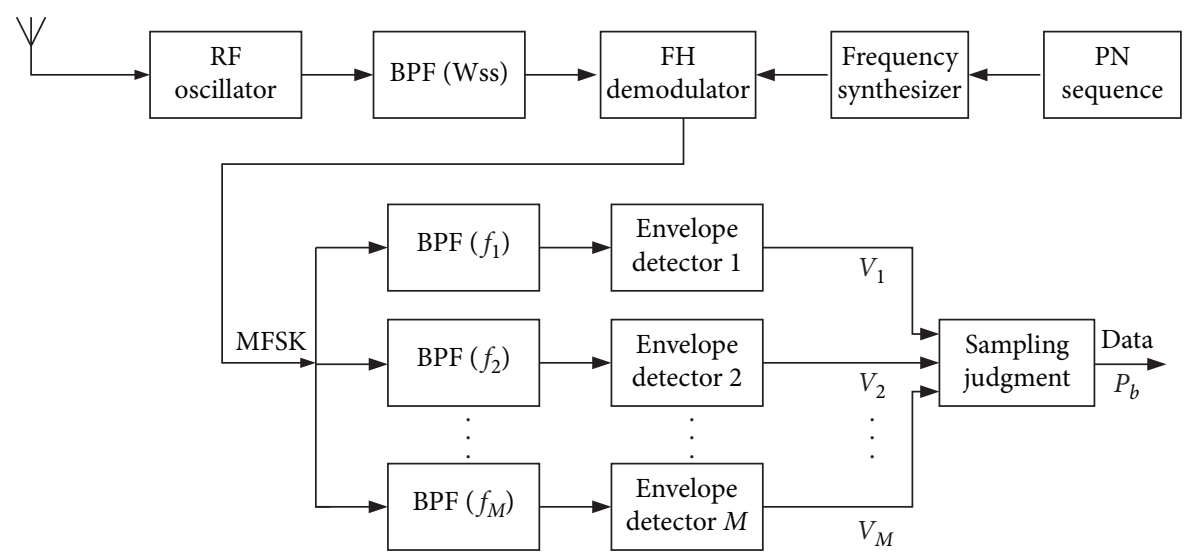

Figure 2: Demodulation model of envelope detection method for FH/MFSK signal.

$$
f\left(V_{1}\right)=\frac{V_{1}}{\sigma^{2}} e^{-\left(V_{1}^{2}+E_{s}\right) / 2 \sigma^{2}} I_{0}\left(\frac{\sqrt{E_{s}} V_{1}}{\sigma^{2}}\right),
$$

where $\sigma^{2}$ represents equivalent noise variance containing variance of external noise and FJ, i.e., $\sigma^{2}=\sigma_{w}^{2}+\sigma_{j}^{2} ; I_{0}(\cdot)$ represents the first class of zero-order modified Bessel function. Other $M-1$ envelope detectors only contain external noise, and their outputs $V_{i}(i=2,3, \ldots, M)$ are Rayleigh distribution with zero mean and variance of $\sigma_{w}^{2}$. The one-dimensional probability density function of $V_{i}$ can be expressed as [15]

$$
f\left(V_{i}\right)=\frac{V_{i}}{\sigma_{w}^{2}} e^{-V_{i}^{2} / 2 \sigma_{w}^{2}} \quad(i=2,3, \ldots, M) .
$$

According to the judging principle of envelope detection method, $V_{i}$ is mutually independent, and there is a fact that the detecting probability of $V_{1}$ is always bigger than that of $V_{i}(i \neq 1)$, i.e., $V_{1}>V_{2}, V_{1}>V_{3}, \ldots, V_{1}>V_{M}$ when sampling judgment makes a correct decision. Thus, the probability of a symbolic decision being correct can be expressed as

$$
\begin{aligned}
P_{r} & =P\left(V_{1}>V_{2}, V_{1}>V_{3}, \ldots, V_{1}>V_{M}\right) \\
& =\int_{0}^{\infty} f\left(V_{1}\right) P\left(V_{2}<V_{1}, V_{3}<V_{1}, \ldots, V_{M}<V_{1} \mid V_{1}\right) \mathrm{d} V_{1} \\
& =\int_{0}^{\infty} f\left(V_{1}\right)\left[\int_{0}^{V_{1}} f\left(V_{1}\right) \mathrm{d} V_{i}\right]^{M-1} \mathrm{~d} V_{1} .
\end{aligned}
$$

The relation between $\operatorname{BER}\left(P_{b}\right)$ and $\operatorname{SER}\left(1-P_{r}\right)$ is given by [16]

$$
P_{b}=\frac{M}{2(M-1)}\left(1-P_{r}\right)
$$

Combining (6)-(9), the formulas of BER are reduced to

$$
P_{b}=\frac{M}{2(M-1)} \sum_{k=1}^{M-1}(-1)^{k+1} C_{M-1}^{k} \frac{\sigma_{w}^{2}}{k \sigma^{2}+\sigma_{w}^{2}} e^{-k E_{s} / 2\left(k \sigma^{2}+\sigma_{w}^{2}\right)} \text {. }
$$

Noting that $m=\log _{2} M$ and $E_{s}=m E_{b}$, the BER of FH/ MFSK system with FNJ is finally expressed as 


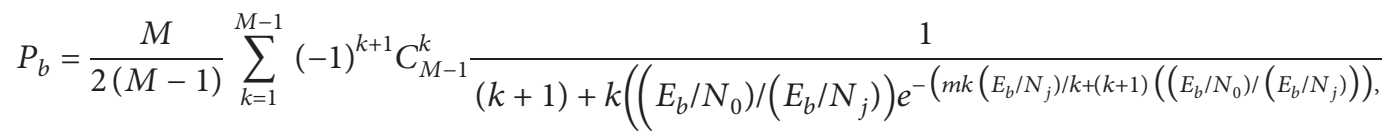

with $E_{b} / N_{0}$ being bit signal-noise ratio and $E_{b} / N_{j}$ being bit signal-interference ratio.

3.2. Modulated Signal of FJ Is Single Tone Signal. When the modulated signal of FJ is single tone signal, called as follower tone jamming (FTJ), its mathematical model can be expressed as

$$
J(t)=\sqrt{\frac{2 E_{j}}{T_{d}}} e^{j 2 \pi f_{n} t}
$$

where $E_{j}$ is the symbol energy of jamming signal.

Making the following notations:

(i) $A$ is the amplitude sum of one-hop signal and corresponding jamming signal when FH signal and jamming signal are simultaneously present.

(ii) $\phi_{\theta-\varphi}$ is the phase difference of one-hop signal and corresponding jamming signal when FH signal and jamming signal are simultaneously present.

Therefore, $A$ can be expressed as

$$
A=\sqrt{E_{s}+E_{j}+2 \sqrt{E_{s} E_{j}} \cos \phi_{\theta-\varphi}} .
$$

Although the phase difference $\phi_{\theta-\varphi}$ has a triangular distribution on a $4 \pi$ interval, $\cos \phi_{\theta-\varphi}$ is uniformly distributed on a $2 \pi$ interval, whose probability distribution is given by [13]

$$
P\left(\cos \phi_{\theta-\varphi} \leq x\right)=1-\frac{1}{\pi} \cos ^{-1} x,|x| \leq 1 .
$$

Same as the case of narrow-band noise, the output of envelope detector 1 contains FJ and external noise. $V_{1}$ is also a generalized Rayleigh distribution with zero mean but variance of $\sigma_{w}^{2}$. The one-dimensional probability density function of $V_{1}$ can be expressed as

$$
f\left(V_{1}\right)=\frac{V_{1}}{\sigma_{w}^{2}} e^{-\left(V_{1}^{2}+A^{2}\right) / 2 \sigma_{w}^{2}} I_{0}\left(\frac{A V_{1}}{\sigma_{w}^{2}}\right) .
$$

The one-dimensional probability density function of other $M-1$ envelope detectors' outputs is the same as (7).

Combining (13)-(15) and (7)-(9), the formulas of BER are reduced to

$$
P_{b}=\frac{M}{2(M-1)} \sum_{k=1}^{M-1}(-1)^{k+1} C_{M-1}^{k} \frac{1}{k+1} e^{-\left(k\left(m E_{b}+E_{j}\right) /(k+1) N_{0}\right)} I_{0}\left(\frac{2 k}{k+1} \sqrt{\frac{m E_{b} E_{j}}{N_{0}^{2}}}\right) .
$$

Noting that interference-signal ratio $\gamma=\left(P_{j} / P_{s}\right)=\left(E_{j} / E_{s}\right)=\left(E_{j} / m E_{b}\right)$, where $P_{j}$ and $P_{s}$ represent the power of jamming signal and signal, respectively, the BER of FH/MFSK system with FTJ is finally expressed as

$$
P_{b}=\frac{M}{2(M-1)} \sum_{k=1}^{M-1}(-1)^{k+1} C_{M-1}^{k} \frac{1}{k+1} e^{-(m k / k+1)(1+\gamma)}\left(E_{b} / N_{0}\right) I_{0}\left(\frac{2 m k}{k+1} \sqrt{\gamma} \frac{E_{b}}{N_{0}}\right)
$$

\section{Numerical Results and Discussion}

In this section, some numerical results of the derived BER formulas with FNJ and FTJ are presented, respectively. Analytical results are obtained from the theoretical BER formulas derived in Section 3. Simulation results are obtained by first generating a sequence of binary data for the desired FH signal. It is then mapped into an M-ary symbol stream, and MFSK modulation is performed. Following that, $\mathrm{FH}$ modulation is performed, and the resultant signal is passed through an AWGN channel with FJ added. At the receiving end, the envelope detection is performed before sampling judgment making a final decision. The number of total frequency points $N$ is set to 32 and frequency interval of $\mathrm{FH}$ is set to $25 \mathrm{kHz}$. The hopping speed is $200 \mathrm{hop} / \mathrm{s}$, and time delay $\Delta t$ is set to zero. A numerical research is performed over the parameters $E_{b} / N_{0}, E_{b} / N_{j}$, and $P_{s} / P_{j}$ at different modulation order $M$.

4.1. FNJ Case. Figure 3 shows BER curves for FH/MFSK system in FNJ case with different $E_{b} / N_{j}$ and $M . E_{b} / N_{j}=\mathrm{INF}$ represents the condition there is no FNJ, and (11) is reduced to (5). The close match between analytical results and simulation results in Figures 3(a)-3(c) verifies the theoretical BER formulas derived in Section 3.1.

It is clear to see that the BER of FH/MFSK system in FNJ case decreases as $E_{b} / N_{0}$ increases. Moreover, the BER increases with the decrease of $E_{b} / N_{j}$ until a peak is reached, which is shown as the black line in Figures 3(a)-3(c). 

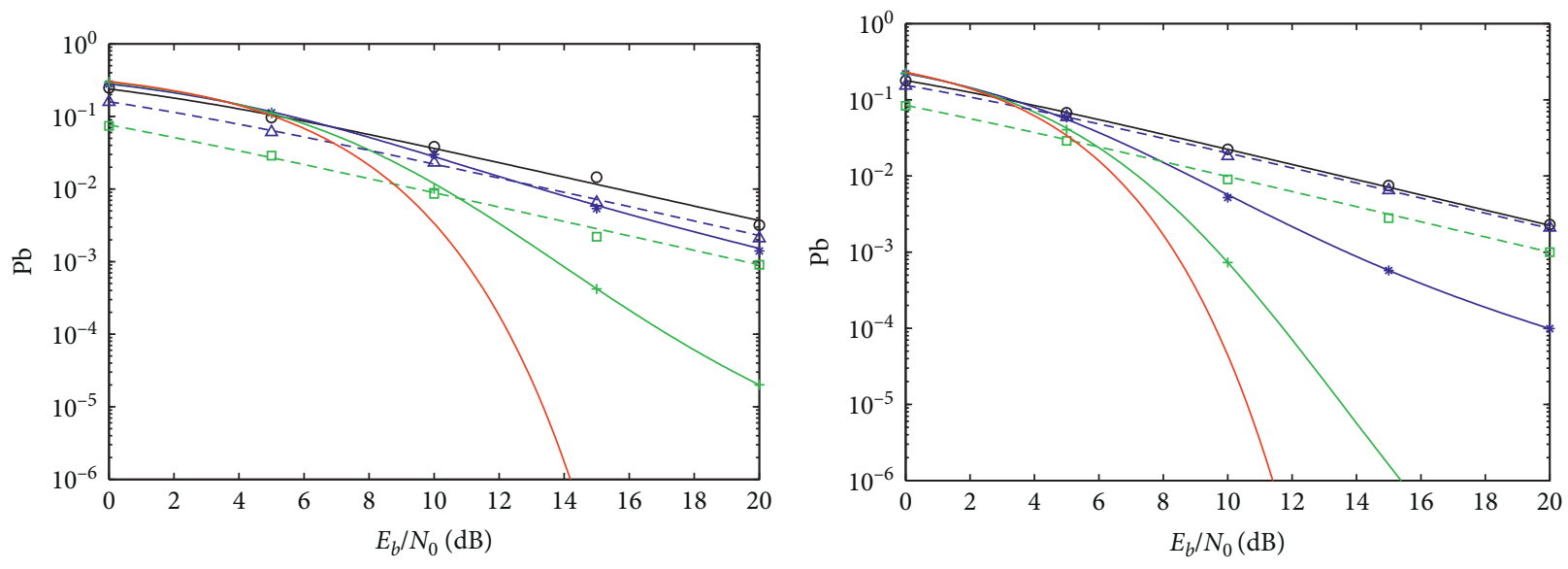

$-E_{b} / N_{j}=0 \mathrm{~dB}$, analytical results

- $E_{b} / N_{j}=0 \mathrm{~dB}$, simulation results

- $E_{b} / N_{j}=5 \mathrm{~dB}$, analytical results

* $E_{b} / N_{j}=5 \mathrm{~dB}$, simulation results

- $E_{b} / N_{j}=10 \mathrm{~dB}$, analytical results

$+E_{b} / N_{j}=10 \mathrm{~dB}$, simulation results

- - $E_{b} / N_{j}=-5 \mathrm{~dB}$, analytical results

$\triangle \quad E_{b} / N_{j}=-5 \mathrm{~dB}$, simulation results

- - $E_{b} / N_{j}=-10 \mathrm{~dB}$, analytical results

- $E_{b} / N_{j}=-10 \mathrm{~dB}$, simulation results

$-E_{b} / N_{j}=$ INF, analytical results

(a)

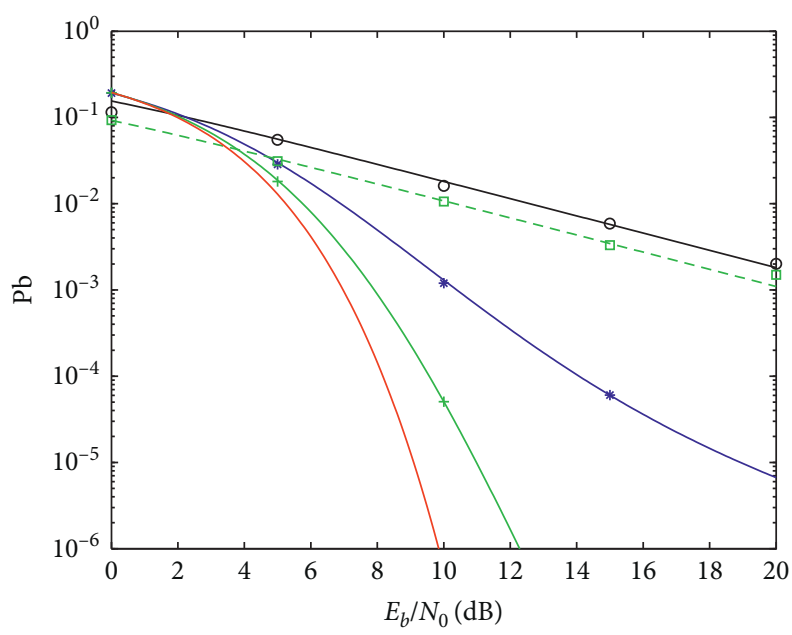

$-E_{b} / N_{j}=5 \mathrm{~dB}$, analytical results

* $\quad E_{b} / N_{j}=5 \mathrm{~dB}$, simulation results

- $E_{b} / N_{j}=-4.77 \mathrm{~dB}$, analytical results

- $E_{b} / N_{j}=-4.77 \mathrm{~dB}$, simulation results

$E_{b} / N_{j}=10 \mathrm{~dB}$, analytical results

$+E_{b} / N_{j}=10 \mathrm{~dB}$, simulation results

- - $E_{b} / N_{j}=-10 \mathrm{~dB}$, analytical results

- $E_{b} / N_{j}=-10 \mathrm{~dB}$, simulation results

$E_{b} / N_{j}=$ INF, analytical results

(c)

Figure 3: BER curves for FH/MFSK system in FNJ case with different $E_{b} / N_{j}$ and $M$. (a) $M=2$. (b) $M=4$. (c) $M=8$. 


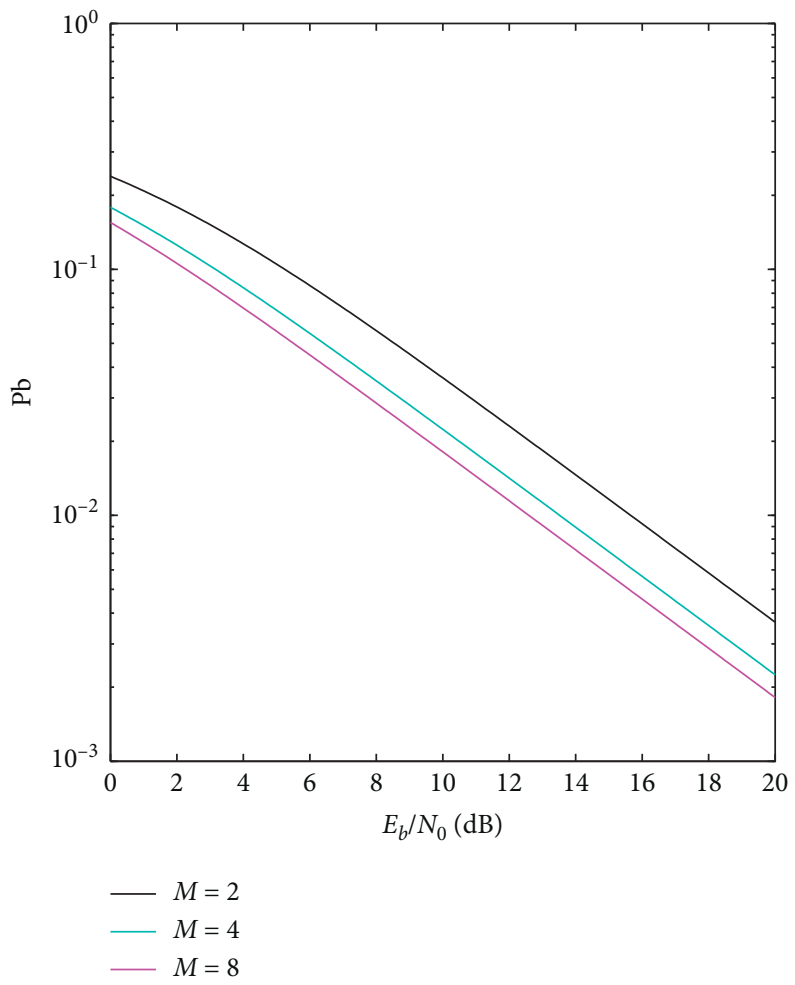

FIGURE 4: BER curves for FH/MFSK system with FNJ in worst case.

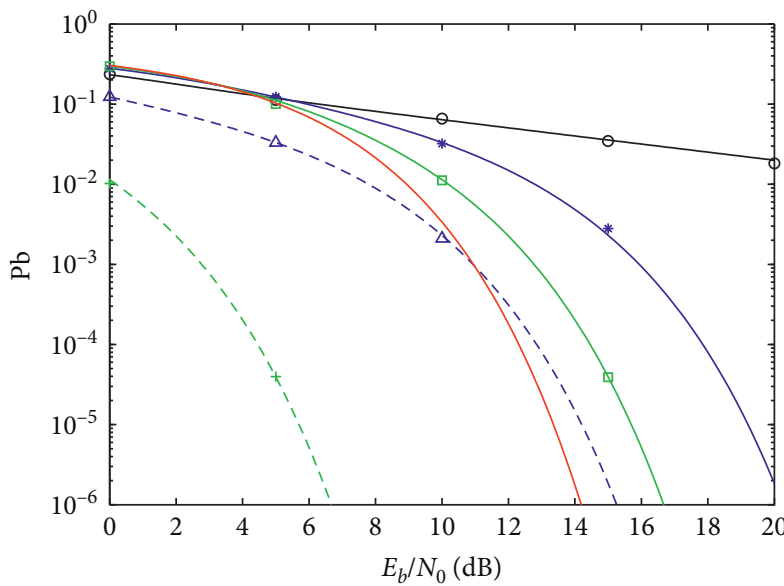

- $P_{s} / P_{j}=0 \mathrm{~dB}$, analytical results

- $P_{s} / P_{j}=0 \mathrm{~dB}$, simulation results

$-P_{s} / P_{j}=5 \mathrm{~dB}$, analytical results

* $\quad P_{s} / P_{j}=5 \mathrm{~dB}$, simulation results

$P_{s} / P_{j}=10 \mathrm{~dB}$, analytical results

ㅁ $\quad P_{s} / P_{j}=10 \mathrm{~dB}$, simulation results

- - $P_{s} / P_{j}=-5 \mathrm{~dB}$, analytical results

$\triangle \quad P_{s} / P_{j}=-5 \mathrm{~dB}$, simulation results

$--P_{s} / P_{j}=-10 \mathrm{~dB}$, analytical results

$+\quad P_{s} / P_{j}=-10 \mathrm{~dB}$, simulation results

$-P_{s} / P_{j}=\mathrm{INF}$, analytical results

(a)

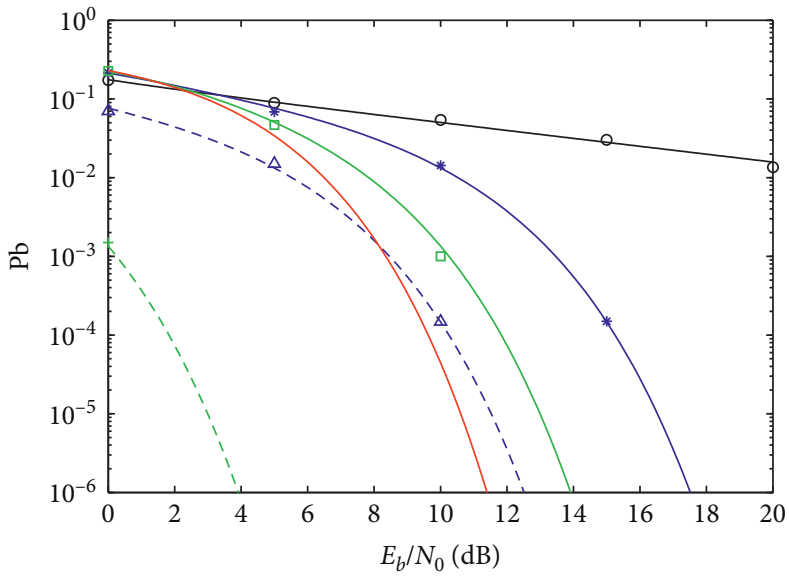

- $P_{s} / P_{j}=0 \mathrm{~dB}$, analytical results

- $P_{s} / P_{j}=0 \mathrm{~dB}$, simulation results

- $P_{s} / P_{j}=5 \mathrm{~dB}$, analytical results

* $\quad P_{s} / P_{j}=5 \mathrm{~dB}$, simulation results

- $P_{s} / P_{j}=10 \mathrm{~dB}$, analytical results

- $\quad P_{s} / P_{j}=10 \mathrm{~dB}$, simulation results

- - $P_{s} / P_{j}=-5 \mathrm{~dB}$, analytical results

$\triangle \quad P_{s} / P_{j}=-5 \mathrm{~dB}$, simulation results

- $P_{s} / P_{j}=-10 \mathrm{~dB}$, analytical results

$+\quad P_{s} / P_{j}=-10 \mathrm{~dB}$, simulation results

$-P_{s} / P_{j}=\mathrm{INF}$, analytical results

(b)

Figure 5: Continued. 


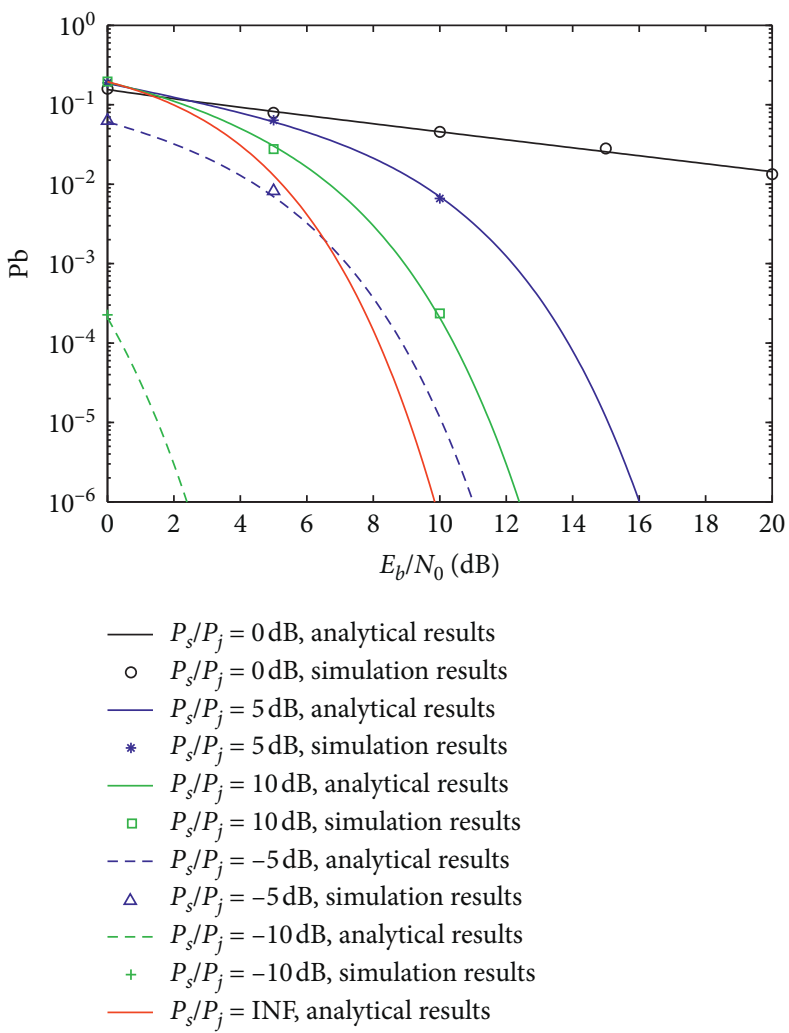

(c)

FIgURE 5: BER curves for FH/MFSK system in FTJ case with different $P_{s} / P_{j}$ and $M$. (a) $M=2$. (b) $M=4$. (c) $M=8$.

Decreasing $E_{b} / N_{j}$ (or increasing the jamming power) exceeding this point will only improves system performance, and the BER decreases. In fact, it can be observed that there is an optimum value of $E_{b} / N_{j}$ which makes BER come to maximum for different $M$. The optimum value of $E_{b} / N_{j}$ depends on the fixed $E_{s} / N_{j}$ (symbol signal-interference ratio), and the relationship can be expressed as

$$
m\left(\frac{E_{b}}{N_{j}}\right)_{\text {opt }}=\frac{E_{s}}{N_{j}}=1 .
$$

Thus, the value conforms to $\left(E_{b} / N_{j}\right)_{\text {opt }}=1 / \log _{2} M$. Corresponding $\left(E_{b} / N_{j}\right)$ are $1(0 \mathrm{~dB}), 1 / 2(-3 \mathrm{~dB})$, and $1 / 3$ $(-4.77 \mathrm{~dB})$ in Figures 3(a)-3(c) individually.

Figure 4 shows BER curves for FH/MFSK system with FNJ in worst case. It describes the BER results in optimum value of $E_{b} / N_{j}$. It can be observed that a higher modulation order $M$ makes a lower BER. The BER of FH/4FSK and FH/ 8 FSK system has a decline of $2 \mathrm{~dB}$ and $3 \mathrm{~dB}$ individually compared with that of FH/2FSK system. Thus, an FH/MFSK system with higher modulation order can improve performance against FNJ.

4.2. FTJ Case. The BER results for FH/MFSK system in FTJ case with different $P_{s} / P_{j}(1 / \gamma)$ and $M$ are shown in Figure 5. $P_{s} / P_{j}=$ INF represents no FTJ as well, and (17) is reduced to
(5). The close match between analytical results and simulation results in Figures 5(a)-5(c) verifies the theoretical BER formulas derived in Section 3.2.

It can be seen that the BER of FH/MFSK system in FTJ case decreases as $E_{b} / N_{0}$ increases similarly. Simultaneously, the BER increases with the decrease of $P_{s} / P_{j}$ until a peak is reached, which is shown as the black line in Figures 5(a)5(c). Decreasing $P_{s} / P_{j}$ (or increasing the jamming power) exceeding this point will only improves system performance as well as the FNJ case, and the BER decreases. Different with FNJ case, the optimum value of $P_{s} / P_{j}$ has nothing to do with the order $M$. Moreover, there is a conclusion that $\left(P_{s} / P_{j}\right)_{\text {opt }}$ is identically equal to $1(0 \mathrm{~dB})$.

Figure 6 shows BER curves for FH/MFSK system with FTJ in worst case, illustrating the BER results in optimum value of $P_{s} / P_{j}$. Similarly, a higher modulation order $M$ makes a lower BER with the same conditions. The BER of $\mathrm{FH} / 4 \mathrm{FSK}$ and FH/8FSK system is reduced by $2 \mathrm{~dB}$ and $3 \mathrm{~dB}$, respectively, compared with that of FH/2FSK system. Thus, an FH/MFSK system with higher modulation order can improve performance against FTJ.

Comparing Figures 4 and 6 , it can be seen that FTJ is more effective to jamming FH/MFSK system than FNJ in worst case, and the latter one will cause a higher BER. Nevertheless, the effectiveness of FTJ will decrease fast when $P_{s} / P_{j}$ is not equal to $0 \mathrm{~dB}$ comparing Figures 3 and 5 . 


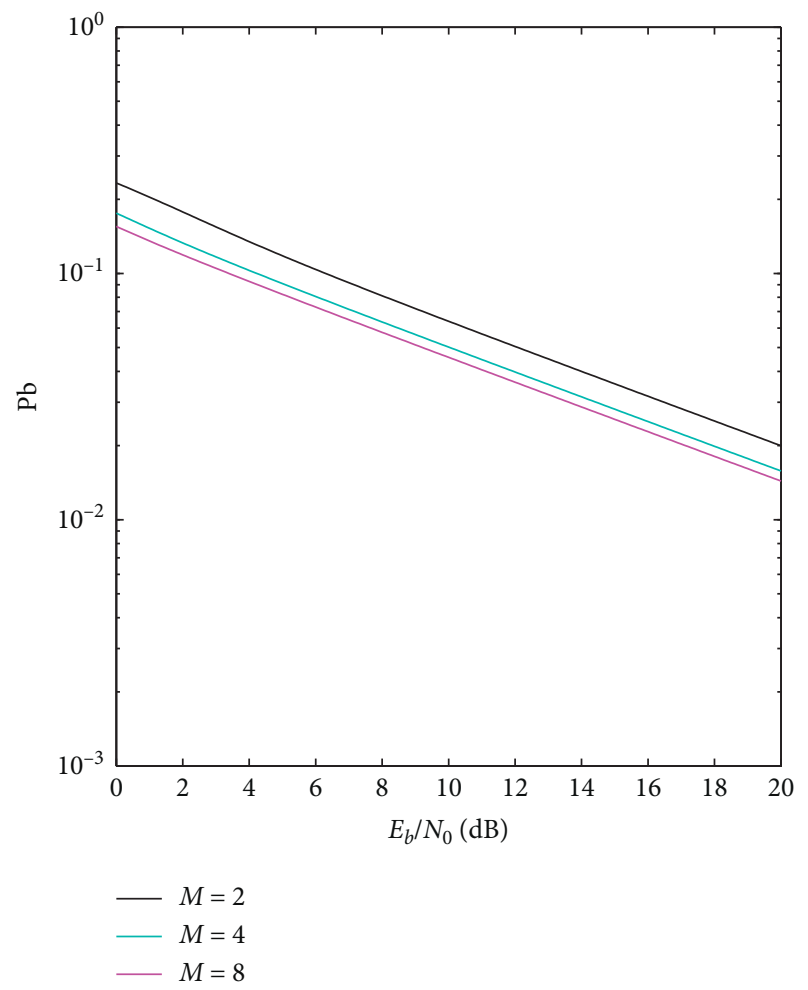

Figure 6: BER curves for FH/MFSK system with FTJ in worst case.

\section{Conclusion}

In this paper, a method of BER analysis with $\mathrm{FJ}$ is proposed for FH/MFSK system. Assuming the wireless channel is an AWGN channel, the BER formulas for different jamming types are derived, respectively, from the point of one-dimensional probability density function of the output signal of the envelope detector by theoretical derivation. Numerical results verify the correctness of the theoretical derivation. Results show that multisystem has a lower BER than binary system, and the BER of FH/4FSK and $\mathrm{FH} / 8 \mathrm{FSK}$ system has a decline of $2 \mathrm{~dB}$ and $3 \mathrm{~dB}$, respectively, compared with $\mathrm{FH} / 2 \mathrm{FSK}$. FNJ is more effective to jamming FH/MFSK system than FTJ in non-worst case. The work in this paper provides an effective means to analyze the BER with FJ, while it is needed to conduct further research for having no regard for the influence of time delay, error correction coding, and convolution interleaving.

\section{Data Availability}

The data used to support the findings of this study are available from the corresponding author upon request.

\section{Conflicts of Interest}

The authors declare that they have no conflicts of interest regarding the publication of this paper.

\section{Acknowledgments}

This study was supported by the Key Pre-Research Fund under Grant no. 9140C87030413JB34001.

\section{References}

[1] F. Yao, Communication Anti-jamming Engineering and Practice, Publishing House of Electronics Industry, Beijing, China, 2nd edition, 2012.

[2] K. S. Marvin, K. O. Jim, A. S. Robert et al., Spread Spectrum Communication Handbook, McGraw-Hill, New York, NY, USA, 2002.

[3] Y.-H. Duan and J. Zhong, "Performance evaluation of multihop FH/MFSK under partial-band jammer," Comunications Technology, vol. 40, no. 12, pp. 7-9, 2007.

[4] J. Zhang, K. C. Teh, and K. H. Li, "Maximum-likelihood FFH/ MFSK receiver over Rayleigh-fading channels with composite effects of MTJ and PBNJ," IEEE Transactions on Communications, vol. 59, no. 3, pp. 675-679, 2011.

[5] L. M. D. Le and K. C. Teh, "Maximum-likelihood FFH/MFSK receiver with $\mathrm{MTJ}$ and $\mathrm{PBNJ}$ over frequency-selective Rayleigh-fading channels plus timing and frequency offsets," in Proceedings of 9th International Conference on Communications and Signal Processing, pp. 1-5, Beijing, China, December 2013.

[6] H. Quan, H. Zhao, and P. Cui, "Anti-jamming frequency hopping system using multiple hopping patterns," Wireless Personal Communications, vol. 81, no. 3, pp. 1159-1176, 2015.

[7] H. Quan, H. Zhao, and P. Cui, "Multi-sequence frequency hopping system and its BER performance analysis over AWGN channel," Journal of Signal Processing, vol. 29, no. 5, pp. 600-606, 2013.

[8] B.-H. Dong, Y. Du, L.-L. Zhou, and J.-Y. Chen, "Performance analysis of broadband frequency sweeping jamming for $\mathrm{FH} /$ MFSK systems," Journal of University of Electronic Science and Technology of China, vol. 38, no. 5, pp. 109-112, 2012.

[9] L. P. Riddle, "Performance of a hybrid spread spectrum system against follower jamming," in Proceedings of IEEE Conference on Military Communications, pp. 420-424, Monterey, CA, USA, September 1990.

[10] A. A. Hassan, W. E. Stark, and J. E. Hershey, "Error rate for optimal follower tone-jamming," IEEE Transactions on Communications, vol. 44, no. 5, pp. 546-548, 1996.

[11] J. Wang and C. Jiang, "Analytical study of FFH systems with square-law diversity combining in the presence of multitone interference," IEEE Transactions on Communications, vol. 48, pp. 1188-1196, 2000.

[12] X. Fan and Z. Tan, "Simulink implementation of frequencyhopping communication system and follower jamming," in Proceedings of 2018 IEEE International Conference on Automation, Electronics and Electrical Engineering, pp. 192-195, Shenyang, China, November 2018.

[13] L. E. Miller, J. S. Lee, R. H. French, and D. J. Torrieri, “Analysis of an antijam FH acquisition scheme," IEEE Transactions on Communications, vol. 40, no. 1, pp. 160-170, 1992.

[14] M. Mei, S. Wang, Y. Qiu, and X. Duan, Frequency Hopping Communications, National Defense Industry Press, Beijing, China, 2005.

[15] H. Zhang and L. Cao, Communication Principle, Science Press, Beijing, China, 2007.

[16] J. G. Proakis, Digital Communications, McGraw-Hill, New York, NY, USA, 4th edition, 2001. 